\title{
From single-file diffusion to two-dimensional cage diffusion and generalization of the totally asymmetric simple exclusion process to higher dimensions
}

\author{
P. M. Centres ${ }^{1, *}$ and S. Bustingorry ${ }^{2}$ \\ ${ }^{1}$ Departamento de Física, Instituto de Física Aplicada, Universidad Nacional de San Luis-CONICET, Chacabuco 917, \\ D5700HHW, San Luis, Argentina \\ ${ }^{2}$ CONICET, Centro Atómico Bariloche, 8400 San Carlos de Bariloche, Río Negro, Argentina
}

(Received 21 September 2015; published 20 January 2016)

\begin{abstract}
A two-dimensional constrained diffusion model is presented and characterized by numerical simulations. The model generalizes the one-dimensional single-file diffusion model by considering a cage diffusion constraint induced by neighboring particles, which is a more stringent condition than volume exclusion. Using numerical simulations we characterize the diffusion process and we particularly show that asymmetric transition probabilities lead to the two-dimensional Kardar-Parisi-Zhang universality class. Therefore, this very simple model effectively generalizes the one-dimensional totally asymmetric simple exclusion process to higher dimensions.
\end{abstract}

DOI: 10.1103/PhysRevE.93.012134

\section{INTRODUCTION}

The relevance of the Kardar-Parisi-Zhang (KPZ) equation [1] for studying many different physical situations has been widely acknowledged during the past two decades. Originally developed for modeling surface growth processes [2], its relevance in many other fields continuously increases, covering turbulence [3], domain-wall motion [4], colloidal suspensions [5], and Macdonald processes in probability theory [6], among many interesting physical, chemical, and mathematical processes. Recent progress on the understanding of KPZ processes has come from its link with random matrix theory $[7,8]$, which provides analytical asymptotic solutions for height distribution functions [8-13]. This analytical progress is largely achieved in $1+1$ dimensions, while in order to test and extend the theoretical concepts to higher dimensions one has to rely on numerical simulations [14-24].

One of the key ideas that promoted theoretical progress on the understanding of the KPZ equation is the formal mapping between the one-dimensional KPZ equation and the totally asymmetric simple exclusion process (TASEP), which can be viewed as a process of strongly interacting biased diffusing particles [25-27]. Within this approach, particles and holes diffusing on a one-dimensional line correspond to positive and negative slopes in a $1+1$ fluctuating interface, providing a direct link between particles density and an interface height profile. In the hydrodynamic limit, the fully biased diffusion of particles leads to nonlinear terms in the diffusion equation, which in the related interface height problem corresponds to a KPZ term [26]. Conversely, the symmetric case, also called single-file diffusion process, belongs to the EdwardsWilkinson (EW) [28] universality class [29-31]. Besides the fact that one-dimensional diffusion can be biased or not, leading to $\mathrm{KPZ}$ or EW processes, it is fairly acknowledged that volume exclusion is a key ingredient that allows mapping growing interfaces onto diffusion processes. This restriction means that two diffusing particles cannot occupy the same site, which guarantees a continuity condition of the fluctuating interface.

*Corresponding author: pcentres@unsl.edu.ar
Remarkably, this fruitful relationship between KPZ and TASEP is mainly restricted to the one-dimensional case, while it is clear that generalizing this relationship to higher dimensions would provide important tools to further explore KPZ and related processes. This is not a trivial step and some attempts have already been reported in recent years. In particular, two recent works can be considered as attempts to extend the TASEP to higher dimensions. On one hand, Tamm, Nechaev, and Majumdar [18] recently proposed a layered zigzag model as a two-dimensional generalization of TASEP and showed that the model belongs to the $2+1 \mathrm{KPZ}$ universality class. On the other hand, Odor and coworkers developed a driven lattice-gas model, which can be mapped onto a two-dimensional surface growth model composed of octahedra belonging to the KPZ universality class [16,17,23].

We pursue the idea that asymmetric diffusion can lead to KPZ processes and propose here a simple two-dimensional diffusion model generalizing the single-file diffusion and TASEP processes to higher dimensions. This generalization not only relies on asymmetric diffusion but goes beyond the simple volume exclusion restriction by identifying the key ingredient for this nontrivial generalization: the cage restriction. We then confirm through numerical simulations that the scaling exponents of this simple two-dimensional diffusion model are in agreement with those of EW and KPZ universality classes for the symmetric and asymmetric cases, respectively.

\section{MODEL AND OBSERVABLES}

We will first describe the model system and define some useful observables. In order to illustrate how we generalize to two dimensions the simple volume exclusion diffusion process we will carry out a construction based on its correspondence with its one-dimensional counterpart. The fundamental observation is that in one dimension the implementation of the volume exclusion principle has a simple but severe consequence: Since a particle cannot diffuse to a site that is already taken by a different particle, this implies, in one dimension, that a particle is constrained to stay always in the cage delimited by its two nearest neighbors, which we 
(a)
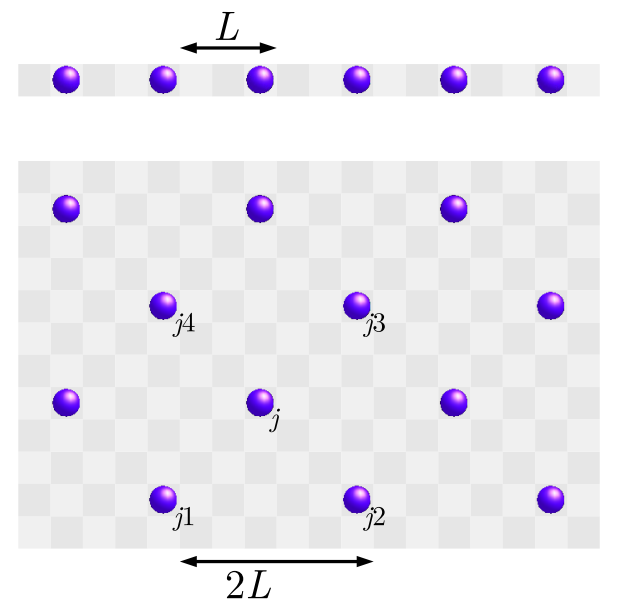

(b)
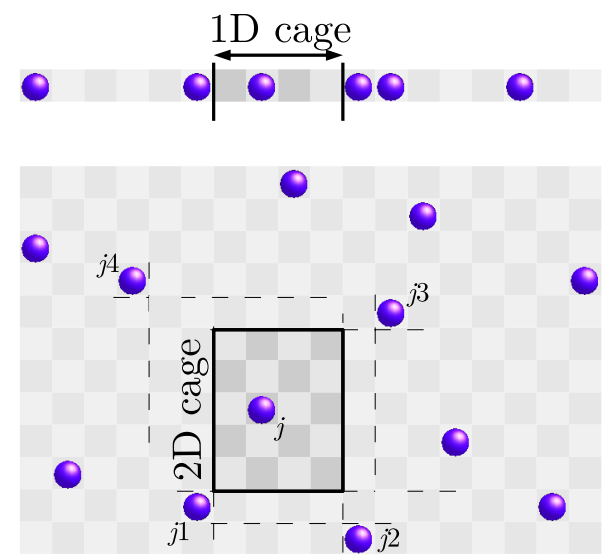

FIG. 1. Schematic representation of the cage diffusion constraint. (a) Initial condition in one and two dimensions. Each particle is located in sites a distance $L$ apart in one dimension, while a sublattice is used in two dimensions where particles form a $\pi / 4$ rotated square lattice of spacing $\sqrt{2} L$. The four initial nearest neighbors of particle $j$ are labeled as $j 1, j 2, j 3$, and $j 4$. (b) The cage diffusion constraint is defined by the position of the nearest neighbors of a particle. In two dimensions, the rectangle indicates the cage where particle $j$ is allowed to diffuse for the given configuration.

shall call the cage diffusion constraint. Therefore, volume exclusion and cage constraint are equivalent in one dimension. This seemingly obvious and inoffensive fact is crucial when thinking on two-dimensional diffusion, where the volume exclusion principle and the cage diffusion constraint are no longer equivalent. The volume exclusion had been naturally extended and widely studied in two dimensions [32-35]. However, the cage diffusion constraint in two dimensions is more restrictive and, to the best of our knowledge, had not been studied.

In order to present our two-dimensional diffusion model based on the cage diffusion constraint, we present in Fig. 1 the one- and two-dimensional discrete realizations used for numerical simulations. Figure 1(a) shows a uniform initial condition. In one dimension $N$ particles are located a distance $L$ apart on a discrete line of unitary spacing. The size of the system is thus given by $M=L N$. The position of particle $j$ are given by $x_{j}=j L$. In two dimensions, particles are initially located in a c $(2 L \times 2 L)$ configuration over the square lattice, thus forming a square lattice of particles separated a distance $\sqrt{2} L$ and rotated $\pi / 4$ with respect to the underlying lattice. The system size, i.e., number of available sites, is now $M^{2}=(L N)^{2}$. The four nearest neighbors of particle $j$ are labeled as $j 1, j 2, j 3$, and $j 4$ in the figure. Figure 1(b) shows the definition of the cage diffusion constraint. Given an arbitrary particle configuration, volume exclusion in one dimension forces each particle to always stay within the indicated cage, delimited by its two nearest neighbors. This can be explicited by stating that the particle position $x_{j}$ is restricted to the condition $x_{j-1}(t)<x_{j}(t)<x_{j+1}(t)$. In two dimensions, volume exclusion would allow particles to explore the system changing its surrounding neighbors over the diffusion process without any confining cage. Therefore, we define the cage diffusion constraint by imposing that each particle is forced to stay within the cage defined by its four initial nearest neighbors. The position of particle $j$ is given by $\mathbf{r}_{j}(t)=x_{j}(t) e_{x}+y_{j}(t) e_{y}$, where $e_{x}$ and $e_{y}$ are unitary vectors on each direction, and it is constrained by those particles identified as nearest neighbors in the initial condition. Therefore, the cage diffusion constraint implies $x_{j}>\left(x_{j 1}, x_{j 4}\right), x_{j}<$ $\left(x_{j 2}, x_{j 3}\right), y_{j}>\left(y_{j 1}, y_{j 2}\right)$, and $y_{j}<\left(y_{j 3}, y_{j 4}\right)$, where $a<(b, c)$ means that $a$ is smaller than the minimum between $b$ and $c$, and $a>(b, c)$ means that $a$ is larger than the maximum between $b$ and $c$. The relevant conditions imposed on particle $j$ by its neighbors positions are indicated as dashed lines in Fig. 1(b), while the cage corresponding to particle $j$ for this configuration is indicated with a rectangle. Notice that the cage restriction is directly linked to a particle label order in the system, which states that once the initial condition is given, each particle conserves full memory of the identity of its nearest-neighbor particles.

Now that we have defined the geometrical diffusion constraint of our model, we need to specify its dynamic rules. We shall consider a diffusion process where a particle in a given site of the square lattice randomly chooses an attempt jump to one of its four neighbor sites. The transition probabilities in the $y$ direction are always symmetric, i.e., $\Gamma_{+y}=\Gamma_{-y}=1 / 4$, while in the $x$ direction we consider a bias field $h$, i.e., $\Gamma_{+x}=1 / 4+h$ and $\Gamma_{-x}=1 / 4-h$. The bias field $h$ is such that $0 \leqslant h \leqslant 1 / 4$, with $h=0$ and $h=1 / 4$ corresponding to the symmetric and totally asymmetric (in the $x$ direction) diffusion cases, respectively. Summarizing, a particle is picked at random, one of its four neighbor sites is chosen with the defined transition probabilities, and if the cage diffusion constrain is satisfied, the particle is moved to the new site. The evolution of the system is given by the evolution of the particle's positions $\mathbf{r}_{j}(t)$, where the unit of time $t$ is given by $N$ jump attempts.

The natural observable to work with when studying diffusion processes is the mean-squared displacement defined by

$$
x^{2}(t)=\frac{1}{N} \sum_{j}\left[\Delta x_{j}(t)\right]^{2}
$$

in the $x$ direction, where $\Delta x_{j}(t)=x_{j}(t)-x_{j}(0)$ gives the displacement with respect to the initial position for the $j$ particle. An equivalent mean-square displacement can be 
defined for the $y$ direction. The mean-square displacement contains valuable information about the diffusion process. For example, if $x^{2}(t) \sim t$ the process is referred to as normal diffusion and the proportionality factor gives the diffusion constant. Deviations from this linear behavior correspond to superdiffusion or subdiffusion whenever the time dependence is stronger or weaker than linear. However, in order to emphasize the relationship between diffusion processes and EW and KPZ universality classes we will characterize the dynamics of the diffusion process using the global roughness of the system. In terms of the fluctuating interface the global roughness measures average height deviations from its mean value. Here, based on the harmonization approximation to single-file diffusion processes [29-31], interface height variables are indeed given by the displacement field $\Delta x_{j}$. Therefore, the global roughness can be defined by

$$
W_{x}^{2}(t)=\frac{1}{N} \sum_{j}\left[\Delta x_{j}(t)-\langle\Delta x\rangle\right]^{2},
$$

where

$$
\langle\Delta x\rangle=\frac{1}{N} \sum_{j} \Delta x_{j}(t)
$$

gives the average center-of-mass displacement at time $t$. In fact, the difference between the mean-square displacement, Eq. (1), and the global roughness, Eq. (2), is that the latter measures the fluctuations with respect to the center of mass of the system. The roughness is expected to present the usual scaling properties observed in the physics of growing interfaces [2,36], described through the scaling relation

$$
W_{x}^{2}(t)=N^{2 \alpha} f\left(\frac{t}{N^{\alpha / \beta}}\right),
$$

with $f(u)$ a scaling function such that

$$
f(u) \sim\left\{\begin{array}{cl}
u^{2 \beta} & \text { for } \quad u \ll 1, \\
\text { const. } & \text { for } \quad u \gg 1 .
\end{array}\right.
$$

Here, $\alpha$ is the roughness exponent characterizing how the roughness asymptotically grows with the system size, $W_{x}(\infty) \sim N^{\alpha}$, and $\beta$ is the growing exponent describing the early time evolution of the global roughness, $W_{x}(t) \sim t^{\beta}$. This also implies the existence of a growing correlation length, $\xi \sim t^{\beta / \alpha}$ [2]. Notice that in our case we are using in the scaling relation the number of diffusing particles as the size of the system, and not the size of the underlying substrate, since the particle index in the diffusion process becomes the spatial dimension in the interface formulation, as can be realized using the harmonization approximation to single-file diffusion processes [29-31].

A useful quantity to unveil the manifestation of timedependent power-law behavior, as contained in Eqs. (4) and (5), is an effective growing exponent defined through

$$
\beta_{\mathrm{eff}}=\frac{1}{2} \frac{\partial \ln W^{2}(t)}{\partial \ln t} .
$$

This effective exponent tends to a constant value for a welldefined power-law behavior and thus can also be indicative of systematic deviations from it.

\section{RESULTS}

In this section we shall present results from numerical simulations of the two-dimensional diffusion model with the cage diffusion constraint. We will first present the symmetric $h=0$ case, then we will focus on the asymmetric case, central for our discussion, and we will finally show some interesting anisotropic effects.

\section{A. Two-dimensional symmetric diffusion}

In order to expose the properties of our diffusion model, we first analyze the behavior of a system with $N=64, L=4$ and without applied bias field, $h=0$. Since our main goal is to describe the diffusion problem in the framework of growing surfaces, we shall first analyze diffusion in terms of the meansquare displacement, its natural variable, and compare it with the global roughness defined in Eq. (2), as shown in Fig. 2.

The mean-squared displacement shows at very small time a lineal dependence on $t$, i.e., a normal diffusion process, where the particles independently move without perceiving its neighboring particles. Then, the system crosses over to a subdiffusive regime signaled by the fact that each particle gradually starts becoming aware of the presence of other particles. More importantly, particles start feeling the cage diffusion constraint, therefore diffusing weaker than linear. Finally, in the third regime observed at long times, each particle is aware of the cage felt by all other particles, therefore indicating that a correlation length has reached the size of the system. This last regime corresponds to the normal diffusion of the center of mass of the system.

The global roughness, similar to the mean-square displacement, shows three regimes with the same crossover times. Within the framework of growing interfaces the independent normal diffusion, subdiffusive, and center-of-mass diffusion regimes would correspond to random deposition of particles on a substrate, correlated growth of the surface, and roughness saturation, respectively. The difference is that in the long-time regime the global roughness gets rid of the center-of-mass diffusion and therefore saturates at a system size dependent

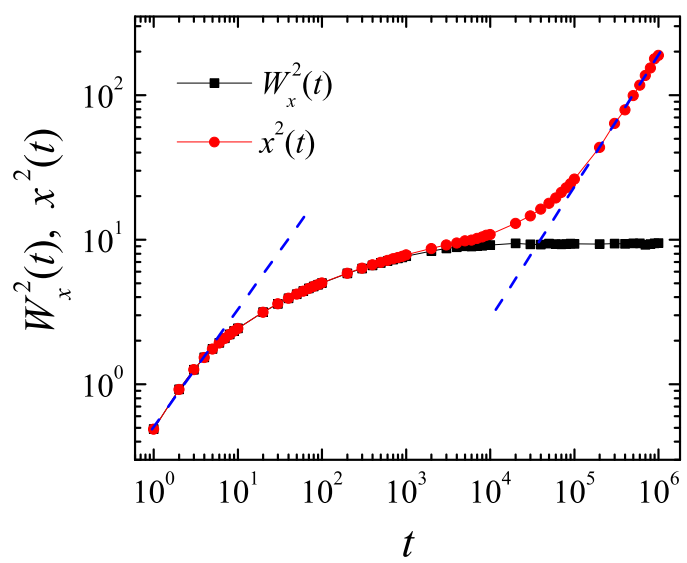

FIG. 2. Comparison between the mean-square displacement $x^{2}(t)$ and the global roughness $W^{2}(t)$ for symmetric diffusion, $h=0$, for a system with $N=64$ and $L=4$. The dashed blue lines indicate normal diffusion regimes. 
value, as indicated in Eq. (4). Consequently, we have shown that the global roughness contains basically the same information than the mean-square displacement regarding the different diffusion regimes.

Based on the relationship between single-file diffusion and one-dimensional EW processes [31], we expect that the roughness behavior presented above for our two-dimensional diffusion model can be described within the two-dimensional EW universality class. Since the upper critical dimension of the EW equation is $d_{c}=2$, it is then expected that, instead of a power-law behavior, the global roughness would logarithmically depend on time and system size as [37]

$$
W^{2}(t)=\frac{D}{2 \pi v} \ln \left[\frac{N}{a} \tilde{f}\left(\frac{v t}{N^{2}}\right)\right],
$$

where $D$ is a white-noise intensity, $v$ is an effective stiffness, $N$ is the system size, and $a$ is a lattice spacing giving a short-range cutoff. In this last equation, $\widetilde{f}(\widetilde{u}) \sim \widetilde{u}^{1 / 2}$ for $\tilde{u} \ll 1$ and $\tilde{f}(\tilde{u}) \sim$ const. for $\tilde{u} \gg 1$, corresponding to $W_{x}^{2} \sim \ln t$ and $W_{x}^{2} \sim \ln N$, respectively. From the information gathered for the one-dimensional case we expect the noise intensity $D$ to be related to the diffusion probabilities and the effective stiffness $v$ to be related to the density of particles, given by $1 / L^{2}[18,31]$.

Figure 3 shows the dynamic global roughness in lin-log scale corresponding to systems with $L=4$ and different number of particles $N$. Since the diffusion is isotropic, the same behavior is observed for $W_{y}^{2}$. One can observe that when increasing $N$ a well-defined logarithmic regime, $W_{x}^{2} \sim \ln t$, is developed, as emphasized by the straight line in Fig. 3. After this subdiffusive regime, a saturation of the global roughness is reached. Furthermore, the saturation values also exhibit a logarithmic dependence on the size of system, as shown in Fig. 4. Therefore, the evolution of the global roughness shows that our two-dimensional symmetric model with the cage diffusion constraint belongs to the Edwards-Wilkinson universality class in its critical dimension.

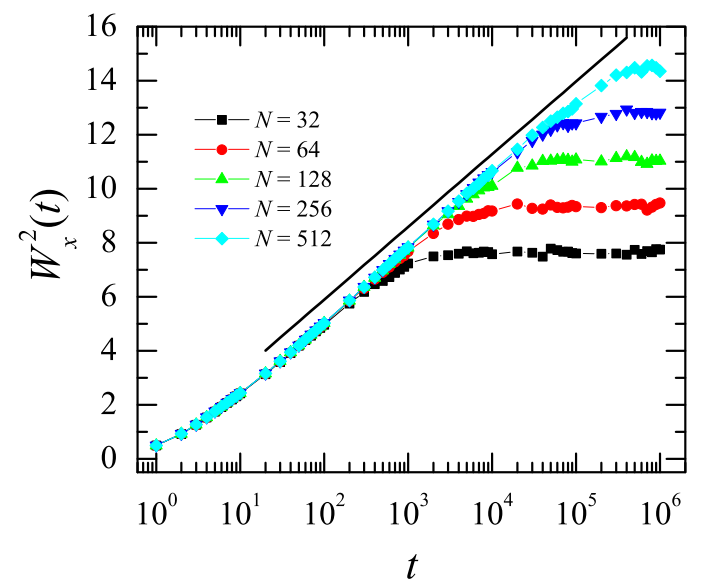

FIG. 3. Evolution of the global roughness corresponding to the symmetric $h=0$ case for a system with average distance $L=4$ and different number of particles $N$, as indicated in the key. The lin-log scale is used to stress the logarithmic dependence on time as indicated by the continuous straight line.

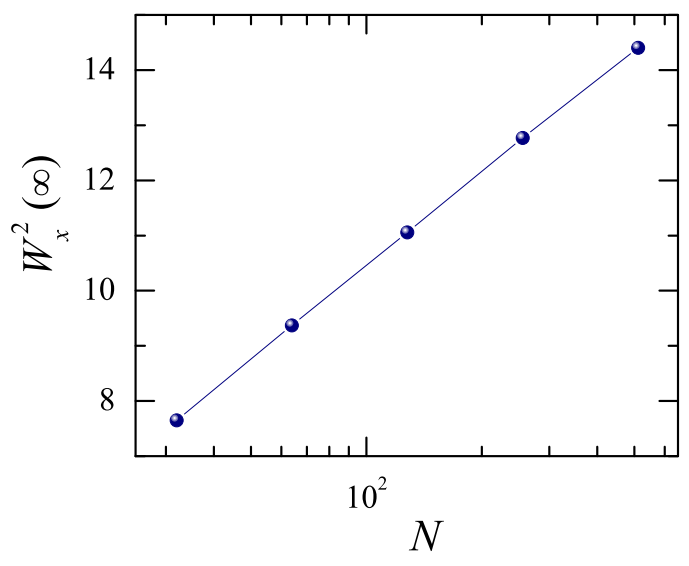

FIG. 4. System size dependence of the saturation value of the global roughness, $W_{x}^{2}(\infty)$, for the symmetric diffusion case and $L=4$. The linear behavior in lin-log scale indicates a logarithmic dependence, $W_{x}^{2}(\infty) \sim \ln N$.

\section{B. Two-dimensional asymmetric diffusion and KPZ processes}

Now we shall show that a finite value of the bias field $h$ induces KPZ characteristics to the two-dimensional diffusion process. Figure 5 shows the change from two-dimensional EW to two-dimensional KPZ behavior when the field is increased. We can observe that regardless the value of the bias field the three regimes are still present, i.e., independent normal diffusion, subdiffusion, and center-of-mass diffusion. On one hand, for $h=0$, the diffusion process corresponds to the two-dimensional Edwards-Wilkinson process, as we showed in the previous section. On the other hand, for $h=$ 0.25 , corresponding to totally asymmetric diffusion in the $x$ direction, we can note that the logarithmic subdiffusive regime is no longer present and instead a purely power-law behavior is observed, as emphasized by the continuous straight line in Fig 5. This power-law behavior for $h=0.25$ and $L=4$ can be observed for system sizes larger than $N=32$. Intermediate values of field allow us to observe the systematic change of behavior between both universality classes. Furthermore,

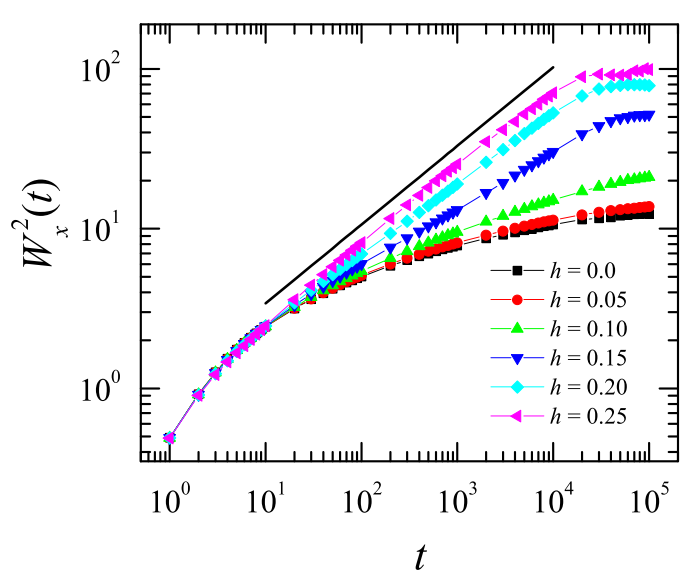

FIG. 5. Evolution of the global roughness $W_{x}^{2}(t)$ for a system with $N=256, L=4$ and different bias field intensities $h$, as indicated. The continuous straight line corresponds to a power-law behavior $W^{2} \sim t^{2 \beta}$ with a growing exponent $\beta=0.247$ (1) corresponding to a two-dimensional KPZ process. 


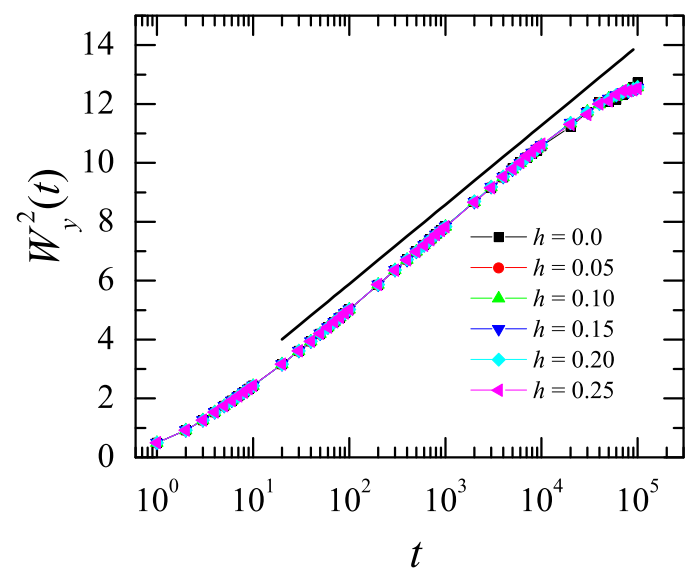

FIG. 6. Evolution of the global roughness $W_{y}^{2}(t)$ in lin-log scale for a system with $N=256, L=4$ and different bias field intensities $h$, as indicated. The continuous straight line corresponds to a logarithmic behavior as expected for a two-dimensional EW process.

measuring the slope of the data between $t=50$ and $t=2000$ for $h=0.25$, we obtain the value $\beta=0.247$ (1) for the growing exponent, in agreement with previous numerical estimates for two-dimensional KPZ processes [16,17,19]. Since a finite field value breaks the symmetry between $x$ and $y$ directions, the behavior of $W_{y}^{2}$ is shown in Fig. 6 for completeness. Fluctuations in the transverse direction to the field can be still characterized by a two-dimensional EW process, $W_{y}^{2}(t) \sim \ln t$, as emphasized by the straight line in Fig. 6.

The change of behavior between two-dimensional EW and two-dimensional KPZ processes driven by the value of $h$ can be further explored using the effective growing exponent $\beta_{\text {eff }}$, as shown in Fig. 7 for the same data presented in Fig. 5. For the symmetric $h=0$ case $\beta_{\text {eff }}$ exhibits a $1 / \ln t$ behavior in accordance with the logarithmic time evolution of $W_{x}^{2}$. As the bias field increases this behavior gradually disappears and finally when $h=0.25$ the effective growing exponent develops a plateau around $\beta_{\text {eff }}=0.24$. For bias field values $0<h<0.25$, the effective growing exponent $\beta_{\text {eff }}$ shows an intermediate behavior that, due to finite size effects, does not develop neither the $1 / \ln t$ nor the plateau limits.

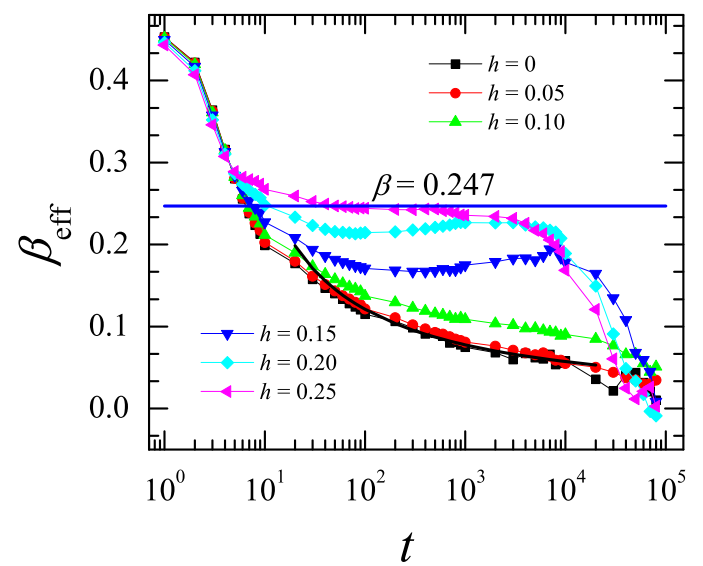

FIG. 7. Effective growing exponent corresponding to the data presented in Fig. 5. The horizontal continuous line corresponds to the $\beta$ value extracted for $h=0.25$ in Fig. 5 .

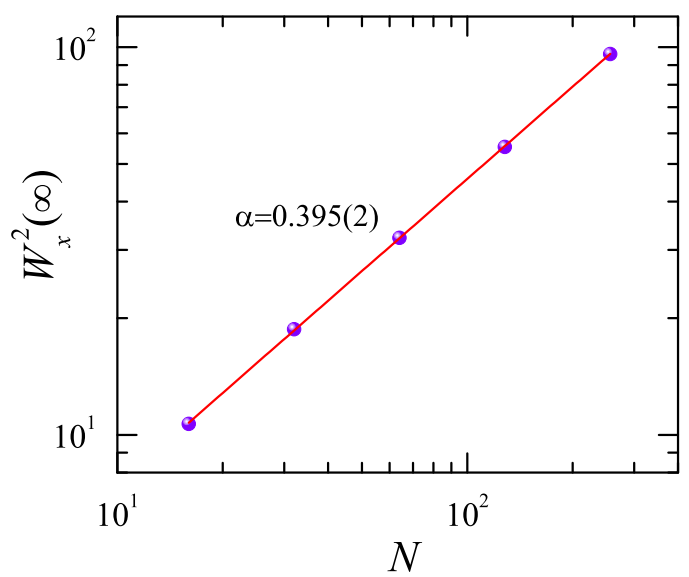

FIG. 8. System size dependence of the saturation value of the global roughness $W_{x}^{2}(\infty)$ for $h=0.25$. The straight line shows a fit with a power-law $W_{x}^{2}(\infty) \sim N^{2 \alpha}$, which gives $\alpha=0.395(2)$, in agreement with a two-dimensional KPZ process.

Finally, Fig. 8 shows, for $h=0.25$, the dependence of the saturation value of the global roughness, $W_{x}^{2}(\infty)$, on the system size $N$. Assuming that values for the roughness saturation are in agreement with the scaling hypothesis given by Eq. (4), the roughness exponent $\alpha$ can be obtained from the slope of the log-log plot, which then gives $\alpha=0.395(2)$. This value agrees with previous numerical results for the twodimensional KPZ universality class $[16,17,19]$. Therefore, our numerical estimates for the growing and roughness exponents, $\beta=0.247$ (1) and $\alpha=0.395(2)$, show that totally asymmetric diffusion with the cage diffusion constraint belongs to the two-dimensional KPZ universality class, i.e., generalizing the TASEP to higher dimensions, which is the central result of the present work.

\section{One-dimensional crossover}

As a by-product of the two-dimensional diffusion process presented here, we show now some results concerning how an anisotropic variation of the size of the system can induce a change on the universality class of the diffusion process. Until now we have been working with isotropic systems whose linear size is given by $M=L N$. We set the average distance $L=4$ and keep the size $M_{x}=L N_{x}$ fixed while the size in the transverse direction is changed as the number of particles $N_{y}$ is reduced, i.e., $M_{y}=L N_{y}$. This would then induce a change between different universality classes through a dimensional crossover.

Figure 9 shows the change of behavior from twodimensional EW to one-dimensional EW behavior when $N_{y}$ is decreased in the symmetric diffusion case $h=0$. For the isotropic $N_{y}=N_{x}$ system the diffusion process belongs to the two-dimensional EW universality class in its critical dimension and presents then a logarithmic time dependence. When $N_{y}$ is reduced, a crossover to one-dimensional EW behavior is observed, as indicated by the appearance of power-law behavior with growing exponent $\beta=1 / 4$ [2]. For intermediate values of $N_{y}$ the presence of a crossover length separating one- and two-dimensional behavior is manifested. 


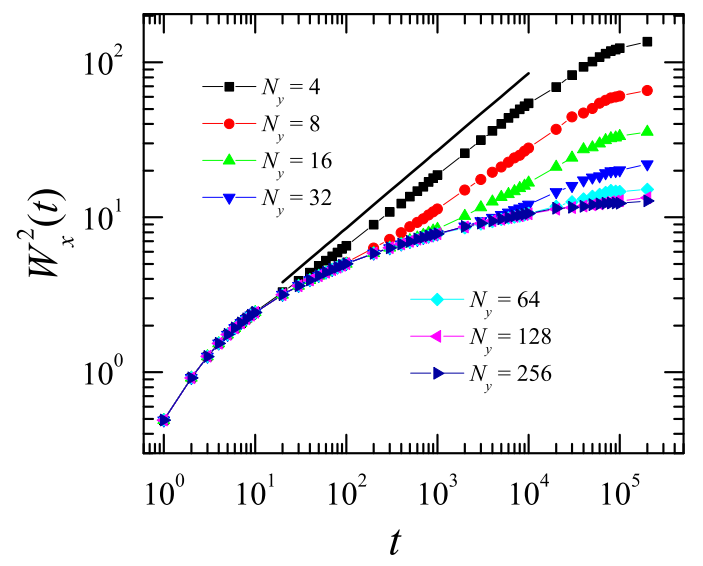

FIG. 9. Evolution of the global roughness $W_{x}^{2}(t)$ for a system with average distance $L=4$ and under anisotropic conditions with $N_{x}=$ 256 and different values of $N_{y}$, as indicated. The symmetric diffusion case $h=0$ is shown, which presents a crossover from logarithmic to power-law behavior when $N_{y}$ is reduced.

When the bias field is turned on at $h=0.25$ in the $x$ direction, one expects a crossover from two-dimensional KPZ to one-dimensional KPZ behavior when $N_{y}$ decreases, as shown in Fig. 10. In this case the power-law behavior changes its growing exponent from the two-dimensional value $\beta=$ 0.247 , as extracted from Fig. 5, to the exact one-dimensional KPZ value $\beta=1 / 3$ [2].

This shows that this simple two-dimensional diffusion model with the cage constraint can be further exploited to study many aspects of the crossover between EW and KPZ processes and its dependence on the dimension of the system.

\section{SUMMARY AND OUTLOOK}

We have presented here a simple model generalizing the TASEP to two dimensions with the aim of recovering a twodimensional KPZ process. We have shown that the key ingredient in this generalization is not volume exclusion but a stringent

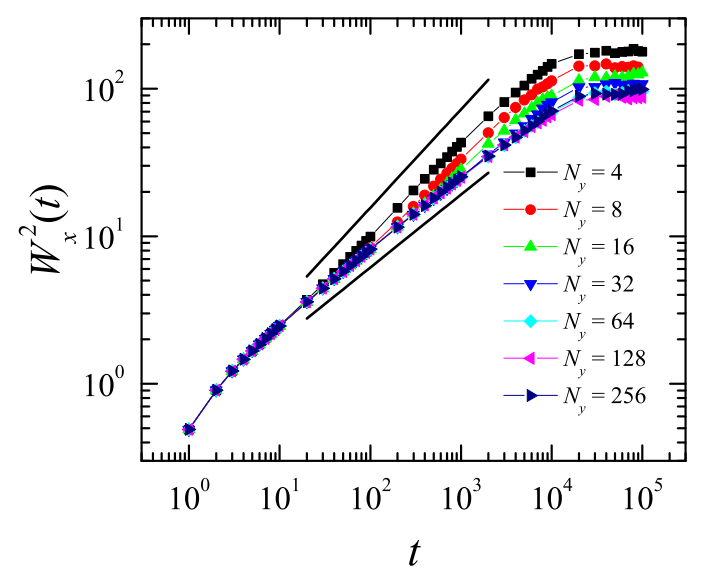

FIG. 10. Evolution of the global roughness $W_{x}^{2}(t)$ for the totally asymmetric case $h=0.25$ for a system with $L=4$ and under anisotropic conditions $N_{x} \neq N_{y}$ with $N_{x}=256$ and different $N_{y}$ values, as indicated. The observed power law, $W_{x}^{2} \sim t^{2 \beta}$, changes its growing exponent from $\beta=0.247$ to $\beta=1 / 3$ when $N_{y}$ decreases. cage diffusion constraint that keeps perfect memory of the surrounding neighbors of each particle over the diffusion process.

In the symmetric diffusion case the model is within the twodimensional EW universality class, therefore presenting an anomalous subdiffusion regime with logarithmic time dependence. When the transition probabilities become asymmetric the logarithmic diffusion regime crosses over to a power-law regime within the two-dimensional KPZ universality class. In the totally asymmetric case our model generalizes the TASEP problem to two dimensions. Generalization to higher dimensions is straightforward. For example, in the threedimensional case a body-centered cubic lattice can be used as initial condition, with the particles in the corner of the cube defining the cage for the subsequent diffusion of the particle in the center of the cube. Both the simple formulation of the model and the fact that moderate system sizes suffice to obtain scaling exponents make this model a good candidate to determine exponents in higher dimensions.

A few further comments are pertinent at this point. First, it has been shown through the relation between one-dimensional single-file diffusion and the EW equation that the effective stiffness associated to the diffusion process is proportional to the density of particles [29-31]. In our two-dimensional model, the slope of the logarithmic time-dependence of the roughness is inversely proportional to the stiffness, as in Eq. (7), and increases with the average separation between particles, thus also supporting that the effective stiffness of the diffusion process is proportional to the density. Furthermore, the effective KPZ parameter $\lambda$ is also expected to depend on density, as shown in Ref. [18] using a coarse-grained hydrodynamic description of a similar two-dimensional model.

Second, though the initial condition we used to define our model and to obtain numerical results seems to be rather arbitrary, any initial condition would work as long as the initial set of neighbors of each particle is kept fixed during the whole diffusion process. For instance, we expect that a random initial condition could also be used. In this case the neighbors could be identified at the very beginning in order to construct and sustain a closed-cage constraint for each particle.

Finally, it is worth stressing that the link between diffusion processes (single-file diffusion, TASEP) and growing interface equations (EW, KPZ), for example using the roughness to characterize the diffusion processes, implies that a well-defined correlation length is growing inside the system of diffusing particles, as it occurs in the growing interface. This correlation length establishes how each particle perceives the fluctuations and surrounding environments of all other particles. Therefore, the cage constraint and its relation to EW and KPZ processes could be relevant in other diffusion processes, like in glass formers or complex fluids, where the origin of growing correlation lengths is still under debate. For example, the strict cage constraint can be relaxed by supplementing the model with a finite probability to jump between neighboring cages, as already treated in one-dimensional systems [38]. This would mimic relaxation processes due to cage diffusion and jumps between different cages, as observed in glass formers [39,40], possibly implying KPZ growing length scales below the average caging timescale.

In conclusion, the two-dimensional diffusion model with cage constraint presented here provides a versatile and easy-to- 
implement model to study different aspects of KPZ processes in higher dimensions.

\section{ACKNOWLEDGMENTS}

This work was partially supported by CONICET (Argentina) under Projects No. PIP11220090100051, No.
PIP11220120100250CO, and No. PIP112-20110100615; Universidad Nacional de San Luis (Argentina) under Project No. 322000; and the ANPCyT (Argentina) under Project No. PICT-2013-1678. The numerical work was done using the BACO parallel cluster located at Instituto de Física Aplicada, Universidad Nacional de San Luis-CONICET, San Luis, Argentina.
[1] M. Kardar, G. Parisi, and Y. C. Zhang, Phys. Rev. Lett. 56, 889 (1986).

[2] A.-L. Barábasi and H. E. Stanley, Fractal Concepts in Surface Growth (Cambridge University Press, Cambridge, England, 1995).

[3] V. V. Anh, N. N. Leonenko, and L. M. Sakhno, J. Stat. Phys. 122, 949 (2006).

[4] K. A. Takeuhchi and M. Sano, J. Stat. Phys. 147, 853 (2012).

[5] P. J. Yunker, M. A. Lohr, T. Still, A. Borodin, D. J. Durian, and A. G. Yodh, Phys. Rev. Lett. 110, 035501 (2013).

[6] A. Borodin and I. Corwin, Probab. Theory Relat. Fields 225, 158 (2014).

[7] V. Dotsenko, Europhys. Lett. 90, 20003 (2010).

[8] M. Prähofer and H. Spohn, Phys. Rev. Lett. 84, 4882 (2000).

[9] K. Johansson, Commun. Math. Phys. 209, 437 (2000).

[10] T. Sasamoto and H. Spohn, Phys. Rev. Lett. 104, 230602 (2010).

[11] G. Amir, I. Corwin, and J. Quastel, Commun. Pure Appl. Math. 64, 466 (2011).

[12] P. Calabrese and P. Le Doussal, Phys. Rev. Lett. 106, 250603 (2011).

[13] T. Imamura and T. Sasamoto, Phys. Rev. Lett. 108, 190603 (2012).

[14] A. Borodin and P. L. Ferrari, Electron. J. Probab. 13, 1380 (2008).

[15] A. Borodin and P. L. Ferrari, J. Stat. Mech. (2009) P02009.

[16] G. Ódor, B. Liedke, and K.-H. Heinig, Phys. Rev. E 79, 021125 (2009).

[17] J. Kelling and G. Ódor, Phys. Rev. E 84, 061150 (2011).

[18] M. Tamm, S. Nechaev, and S. N. Majumdar, J. Phys. A 44, 012002 (2011).

[19] T. Halpin-Healy, Phys. Rev. Lett. 109, 170602 (2012).
[20] T. J. Oliveira, S. G. Alves, and S. C. Ferreira, Phys. Rev. E 87, 040102 (2013).

[21] A. Pagnani and G. Parisi, Phys. Rev. E 87, 010102 (2013).

[22] S. G. Alves, T. J. Oliveira, and S. C. Ferreira, Phys. Rev. E 90, 020103 (2014).

[23] G. Ódor, J. Kelling, and S. Gemming, Phys. Rev. E 89, 032146 (2014).

[24] A. Pagnani and G. Parisi, Phys. Rev. E 92, 010101 (2015).

[25] B. Derrida, Phys. Rep. 301, 65 (1998).

[26] T. Kriecherbauer and J. Krug, J. Phys. A 43, 403001 (2010).

[27] J. Quastel and H. Spohn, J. Stat. Phys. 160, 965 (2015).

[28] S. F. Edwards and D. R. Wilkinson, Proc. R. Soc. A 381, 17 (1982).

[29] S. N. Majumdar and M. Barma, Phys. Rev. B 44, 5306 (1991).

[30] L. Lizana, T. Ambjörnsson, A. Taloni, E. Barkai, and M. A. Lomholt, Phys. Rev. E 81, 051118 (2010).

[31] P. M. Centres and S. Bustingorry, Phys. Rev. E 81, 061101 (2010).

[32] H. van Beijeren and R. Kutner, Phys. Rev. Lett. 55, 238 (1985).

[33] H. van Beijeren and R. Kutner, J. Stat. Phys. 49, 1043 (1987).

[34] J. Jäckie, K. Froböse, and D. Knödler, J. Stat. Phys. 63, 249 (1991).

[35] D. Rostkier-Edelstein and S. Efrima, J. Chem. Phys. 96, 7144 (1992).

[36] F. Family and T. Vicsek, J. Phys. A 18, L75 (1985).

[37] T. Nattermann and L.-H. Tang, Phys. Rev. A 45, 7156 (1992).

[38] D. Lucena, D. V. Tkachenko, K. Nelissen, V. R. Misko, W. P. Ferreira, G. A. Farias, and F. M. Peeters, Phys. Rev. E 85, 031147 (2012).

[39] R. Pastore, A. Coniglio, and M. P. Ciamarra, Soft Matter 10, 5724 (2014).

[40] R. Pastore, A. Coniglio, and M. P. Ciamarra, Soft Matter 11, 622 (2015) 